\title{
Balance de resultados en la asignatura Electrónica y Automática (Grado de Mecánica) con la incorporación de metodologías activas de aprendizaje
}

\section{J. Ernesto Solanes y Luis Gracia}

Departamento de Ingeniería de Sistemas y Automática, Universitat Politècnica de València

\begin{abstract}
It is well known that active models of teaching make the student learning more efficient than with the lecture model of teaching. However, the attachment to the lecture model of teaching makes both students and teachers very reluctant to drastically change to any active model of teaching, especially in subjects with high theoretical content and in first grade courses. For this reason, this article proposes to combine the lecture model of teaching with active methods of teaching in order to improve the teaching-learning process of first grade students. The article describes in detail a case of application for the subject of Electronics and Automation of the second year of the Mechanical Engineering Degree. In addition, a comparison of the academic results obtained with the same teaching staff in the 2018/2019 academic year, using the traditional lecture model of teaching, and the 2019/2020 academic year, combining the lecture model of teaching with active methods, is shown and analyzed. Moreover, the article shows the student opinions about their experience with the subject in the 2019/2020 academic year.
\end{abstract}

Keywords: Activating methods, automation, mechanical degree

\section{Resumen}

Es bien sabido que las modalidades pedagógicas que fomentan que el alumno sea protagonista de su proceso de aprendizaje son más eficaces. Sin embargo, el arraigo hacia la modalidad de clase magistral hace que tanto alumnos como profesores sean muy reacios a cambiar drásticamente a un modelo activo, sobretodo en asignaturas con alto grado teórico y de primeros cursos de grado. Es por ello que este artículo propone combinar la modalidad de clase magistral con metodologías activas de cara a mejorar el proceso de enseñanza-aprendizaje en alumnos de primeros cursos de grado. El artículo describe con detalle un caso de aplicación en la asignatura de Electrónica y Automática de segundo curso de Grado de Ingeniería Mecánica. Además, se muestra una comparativa de los resultados académicos obtenidos con el mismo profesorado en los cursos 2018/2019, con la modalidad tradicional de clase magistral, y el 2019/2020, con la propuesta de este trabajo. Por último, el artículo expone la opinión que han transmitido los alumnos acerca de su experiencia con la asignatura en el curso 2019/2020.

Keywords: Metodologías activas, automática, grado de mecánica. 


\section{Introducción}

En la actualidad, existen varias alternativas al modelo tradicional de clase magistral. Por ejemplo, la modalidad flipped classroom o clase inversa, (Olaizola 2014; Esquivel-Gámez 2014), en la cual los alumnos tienen que adquirir una parte de los conocimientos antes de ir al aula (trabajo en casa), mientras que el aula sirve para afianzar la comprensión de los conocimientos adquiridos en casa mediante un proceso de aprendizaje activo (resolución de problemas, actividades, trabajos en grupo, etc.). Otra de las modalidades que ha cobrado mucha relevancia en los últimos años debido al crecimiento de las Tecnologías de la Información y la Comunicación, (ISTE 1992; Sancho 1995; Martínez Costa, Formoso Barro y Sanjuán Pérez 2017), es la denominada trabajo autónomo, trabajo flexible o estudio a distancia, (Cervera 1999; Gómez Paternina 2009; Cerda y Saiz 2015). En este caso, el profesor guía al alumno en su proceso de auto-aprendizaje proporcionándole herramientas, materiales y recursos que le permitan avanzar en su proceso.

Así mismo, se dispone de una amplia variedad de metodologías pedagógicas enfocadas a mejorar el proceso de aprendizaje del alumnado: el método de Resolución de Ejercicios/Problemas, basada en que el equipo docente proponga actividades/problemas a ser desarrollados por el conjunto de alumnos, aplicando los conceptos fundamentales vistos con anterioridad, (Costa y Moreira 2001); el método del Estudio de Casos, que trata de que los alumnos se enfrenten a situaciones específicas que plantean problemas que necesitan soluciones (Merriam 1998); método de Aprendizaje Basado en Problemas, que trata que los alumnos participen de forma constante en el aprendizaje, adquiriendo habilidades y aptitudes a partir de situaciones de la vida real que los profesores les proponen y, a diferencia del caso del método del Estudio de Casos, no requiere unos conocimientos previos antes de enfrentarse al problema, (Barrows 1986); el método del Aprendizaje Cooperativo, basado en el trabajo en grupo de forma que cada alumno mejore su proceso de aprendizaje individual y el del resto de componentes del grupo, (Johnson y D.W 1994).

Sin embargo, es muy difícil prescindir totalmente del modelo tradicional de clase magistral debido a que, tanto los profesores como los alumnos, están muy acostumbrados a dicho modelo, (SánchezVera y col. 2018). Esto es más evidente en asignaturas de alto contenido teórico impartidas en los primeros cursos de grado. Además, hay que tener en cuenta el factor de la masificación de alumnos en aulas de los primeros cursos de grado, problema que hace que muchos profesores sean reacios a cambiar a modalidades activas de enseñanza.

Dicho esto, es evidente que el modelo de clase magistral presenta varias desventajas, (Propuestas para la Renovación de las Metodologías Educativas en la Universidad 2006). Por ejemplo, la pasividad de los alumnos en el aula, mas centrados en adquirir apuntes que en entender los conceptos transmitidos por el profesor, perjudica a su motivación e interés por la asignatura. El profesor por su parte, no es consciente de cómo están evolucionando los alumnos a medida que avanza el curso, y solamente tiene como evidencia de éxito o fracaso del proceso de enseñanza-aprendizaje las notas de las evaluaciones realizadas a lo largo de la asignatura.

Atendiendo a los problemas que surgen al incorporar de forma radical una modalidad distinta a la de clase magistral en clases masificadas de primeros cursos de grado, y a los beneficios que muchos autores reportan al incorporar metodologías activas en sus asignaturas, el presente trabajo propone combinar la modalidad de clase magistral con varias metodologías activas en la asignatura Electrónica y Automática del segundo curso del Grado de Ingeniería Mecánica impartido en la Escuela Politécnica Superior de Alcoy (EPSA) de la Universitat Politècnica de València. Se trata de una asignatura troncal de 4,5 ECTS (22,5 horas de teoría de aula, 7,5 horas de prácticas en el aula y 15 horas de prácticas en laboratorio, repartidas en 15 semanas lectivas). La media de

2020, Universitat Politècnica de València

Congreso IN-RED (2020) 
alumnos por año es de 100 alumnos, repartidos en dos grupos de teoría (70 alumnos un grupo y 30 alumnos el otro grupo), y 4 grupos de prácticas. Debido a que esta asignatura tiene dos temáticas muy diferenciadas, por una parte Electrónica y por otra parte Automática, cada una de ellas impartida y gestionada por diferentes departamentos, se decide incorporar la innovación solamente en la parte de Automática (12 horas de teoría de aula, 4 horas de prácticas en el aula y 8 horas de prácticas de laboratorio, repartidas en 8 semanas lectivas).

Hasta el curso 2018/2019, inclusive, la modalidad pedagógica utilizada en dicha asignatura fue fundamentalmente la de clase magistral. En el caso de la temática de Automática, las prácticas de laboratorio se realizaban utilizando el software Matlab/Simulink para la simulación de modelos dinámicos y control de sistemas. Durante las clases magistrales, los profesores dedicaban gran parte del tiempo a transmitir información y resolver problemas "tipo" en la pizarra, mientras los alumnos tomaban apuntes y preguntaban dudas.

Analizando un poco la temática de automática impartida en la asignatura, se determina que tiene dos problemas fundamentales: tiene un elevado contenido teórico y abstracto, sobretodo en los conceptos de modelado y control de sistemas; su "necesidad" de cara al futuro laboral no es comprendida por parte de los alumnos de este grado. Prueba de ello son las siguientes afirmaciones extraídas de conversaciones con los alumnos en el curso académico 2018/2019:

"En los inicios de esta asignatura estaba bastante asustado, ya que no tenía conocimientos previos de automática y en las clases teóricas no acababa de relacionar los conceptos con algo real, [...]".

"[...] durante las primeras clases no entendía muy bien el significado de la asignatura ni la utilidad de la misma.".

Es evidente que estos problemas desembocan en una pérdida de interés y motivación por parte del alumnado y, por consiguiente, peores resultados académicos.

Las metodologías activas propuestas en este trabajo, desarrolladas y aplicadas en el curso académico 2019/2020, han sido las siguientes:

- Motivación del alumnado basada en realidad aumentada: se introduce una actividad de dinamización del aula basada en la tecnología de realidad aumentada, (Gattullo y col. 2019), de cara a captar la atención de los alumnos y motivarles a través de la experiencia con el uso de una nueva tecnología.

- Método de Resolución de Ejercicios/Problemas (REP): durante las clases magistrales se proponen varios ejercicios/problemas, que son resueltos de forma individual/por equipos y algunos de ellos se presentan en forma de juego competitivo.

- Método de Aprendizaje Basado en Problemas (ABP): se introduce el problema de identificar el modelo de un prototipo de motor de corriente continua y diseñar un regulador para controlar su velocidad.

- Socialización y motivación del alumnado a través de foros de debate: establecimiento de un foro de debate donde el profesor incorpora temas relacionados con la asignatura y actúa de moderador, mientras que los alumnos establecen opiniones y nuevas informaciones al respecto de los temas discutidos. 
El balance de resultados se ha realizado comparando los resultados académicos obtenidos en los cursos 2018/2019 y 2019/2020, atendiendo a que en estos cursos los profesores que han impartido las clases han sido los mismos y solamente ha cambiado la incorporación de las metodologías activas propuestas. Además, también se muestra un análisis de las opiniones que han mostrado los alumnos en los distintos foros de debate de la asignatura de cara a determinar el impacto de las metodologías activas incorporadas.

El contenido del artículo se estructura de la siguiente manera: la Sección 2, introduce el objetivo general del artículo y los objetivos específicos; la Sección 3 describe los materiales desarrollados y metodologías activas propuestas; la Sección 4 presenta los resultados obtenidos con la propuesta realizada; y finalmente la Sección 5 expone las conclusiones obtenidas.

\section{Objetivos}

El objetivo principal del presente trabajo es el de presentar el balance de resultados y conclusiones extraídas en la aplicación de metodologías activas combinadas con la modalidad clase magistral a la asignatura Electrónica y Automática impartida en $2^{\circ}$ curso del Grado de Mecánica, de modo que pueda animar a otros docentes de asignaturas similares de distintas áreas a ir de forma gradual incorporando metodologías activas en sus clases. Los objetivos específicos son los siguientes:

1.- Mejorar el proceso enseñanza-aprendizaje con la incorporación de metodologías activas.

2.- Evaluar la adecuación de las metodologías activas propuestas.

3.- Analizar el impacto sobre los resultados académicos de los alumnos.

4.- Analizar la información aportada por los alumnos a través de su opinión en foros de debate.

\section{Desarrollo de la innovación}

\subsection{Materiales desarrollados}

\subsubsection{Motivación del alumnado basada en realidad aumentada}

Si bien los medios audiovisuales son los más utilizados en las fases de introducción y motivación de las materias impartidas, es bien sabido que los métodos capaces de establecer curiosidad e interacción son los más efectivos a la hora de motivar.

Con este convencimiento, los autores del presente artículo desarrollaron una actividad para dinamizar el aula basada en realidad aumentada utilizando las gafas Microsoft ${ }^{\circledR}$ HoloLens como dispositivo de interacción. 


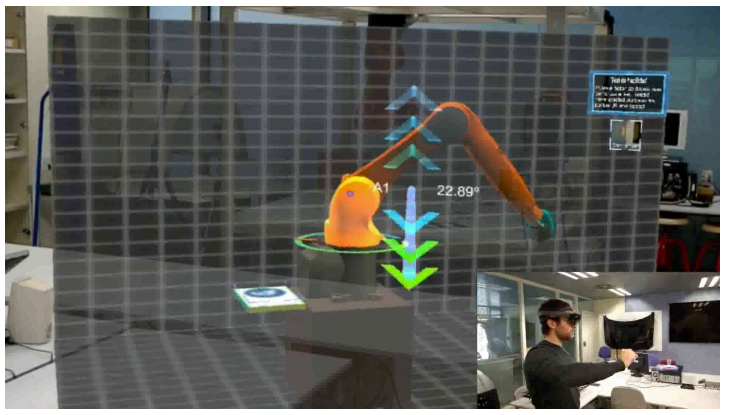

(a)

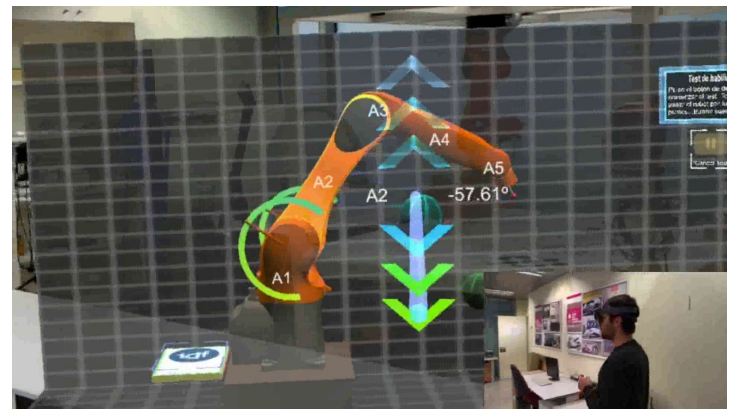

(b)

Fig. 1: Aplicación de realidad amentada utilizada como actividad de dinamización del aula.

La aplicación trata de un robot industrial de 6 grados de libertad que se proyecta en un lugar fijo en el aula (ver Fig. 1) ${ }^{1}$. El alumno puede mover cada uno de los ejes del robot mediante gestos o mediante órdenes de voz. Para poder mover un eje mediante gestos, el alumno selecciona el eje del robot con la mirada, momento en el cuál le aparece un indicador de movimiento que es capaz de controlarlo con sus dedos. Del mismo modo, si decide controlar el eje del robot utilizando la voz, solamente ha de mencionar en alto (en inglés) que eje quiere mover y, en el momento que aparezca el indicador de movimiento, hacia qué dirección quiere moverlo (positivo o negativo).

Mientras un alumno está interactuando con la aplicación, el resto está visualizando lo que éste está viendo en directo por el proyector del aula. De esta forma, todos ellos están pendientes de la actividad y se despierta su interés en probarla.

El objetivo final de esta actividad es el de que los alumnos aprendan de una forma interactiva, los conceptos de sistema, acciones de control, respuesta del sistema y tecnologías emergentes, entre otras.

\subsubsection{Método de Resolución de Ejercicios/Problemas}

Además de los ejercicios resueltos en clase por el profesor, se decide preparar dos actividades de cara a fomentar la participación de los alumnos en el aula:

1. El juego: la actividad consiste en resolver 5 cuestiones y 1 problema en un plazo máximo de 45 minutos. Los alumnos disponen de todo el material de clase (apuntes, diapositivas, internet, etc.). Además pueden colaborar entre ellos. El primer alumno que acabe la actividad y la resuelva correctamente, obtendrá una recompensa. El profesor, en este caso, no puede resolver las dudas de los alumnos durante los 45 minutos de duración de la actividad.

2. Actividad individual: la actividad consiste en resolver 2 ejercicios similares a los realizados por el profesor en anteriores sesiones de clase. En este caso, el alumno no dispone del material de clase y ha de trabajar de forma individual. El profesor puede aclarar dudas. Al acabar la

\footnotetext{
${ }^{1}$ De cara a cumplir con la ley de protección de datos, se ha decidido mostrar la actividad diseñada fuera del aula y con personal de investigación que ha dado su permiso para la difusión de su imagen.
} 
actividad, dos voluntarios resuelven los ejercicios y pueden ser ayudados tanto por el profesor como por los propios compañeros de clase.

Estas actividades tienen dos propósitos: en primer lugar, que el alumno sea consciente del estado de su proceso de aprendizaje a lo largo del curso, de cara a que pueda actuar en consecuencia; que el profesor sea capaz de determinar en qué estado del proceso de aprendizaje se encuentra la mayoría de alumnos, pudiéndose adaptar y dedicar más tiempo a aquellos aspectos que no hayan sido entendidos.

\subsubsection{Método de Aprendizaje Basado en Problemas}

Con el firme convencimiento de los autores de que no hay mejor aprendizaje que el que se alcanza cuando uno se enfrenta a un problema real, se decide cambiar las prácticas realizadas hasta el curso 2018/2019 e introducir el método ABP en prácticas.

El problema a resolver por los alumnos, trabajando en equipos de 4 componentes, es el siguiente: identificación del modelo y control de la velocidad de un motor de corriente continua. Para ello, disponen de un prototipo de motor de corriente continua real (ver Fig. 2(a)) y 6 objetivos a alcanzar:

1. Obtener de forma experimental la función de transferencia del prototipo Motor de Corriente Continua del laboratorio.

2. Simular el comportamiento de la función de transferencia ante entrada escalón.

3. Diseñar un control PID utilizando el método en lazo abierto de Ziegler-Nichols.

4. Simular el comportamiento en lazo cerrado con el controlador PID diseñado.

5. Obtener la respuesta del sistema real en lazo cerrado con el controlador PID diseñado sin perturbaciones.

6. Obtener la respuesta del sistema real en lazo cerrado con el controlador PID diseñado con perturbaciones: análisis de la robustez del controlador.

El software necesario para interactuar con el prototipo motor de corriente continua se ha realizado con las plataformas Matlab y Labview. En cuanto al hardware, el prototipo dispone de una tarjeta Labjack con comunicación serie que actúa de interfaz entre el PC del alumno y el prototipo.

De cara a facilitar la comprensión de los distintos elementos software/hardware por parte del alumnado, se establece que toda la documentación esté disponible en la PoliformaT utilizando la herramienta LESSONS (ver Fig. 2). El motivo principal es que con esta herramienta es posible establecer una estructura documentada y con actividades que los alumnos han de realizar y, por consiguiente, que se sientan guiados en todo momento. ${ }^{2}$

Finalmente, los alumnos han de entregar un informe con el detalle de todas las tareas realizadas para alcanzar cada uno de los objetivos, con los resultados alcanzados y las conclusiones extraídas.

\footnotetext{
${ }^{2}$ Notar que los alumnos a los que va dirigida esta actividad son de primeros cursos de grado, por lo que hay que tener presente qué competencias se les puede exigir (Proyecto de Competencias Transversales del la Universitat Politècnica de València 2015).
} 


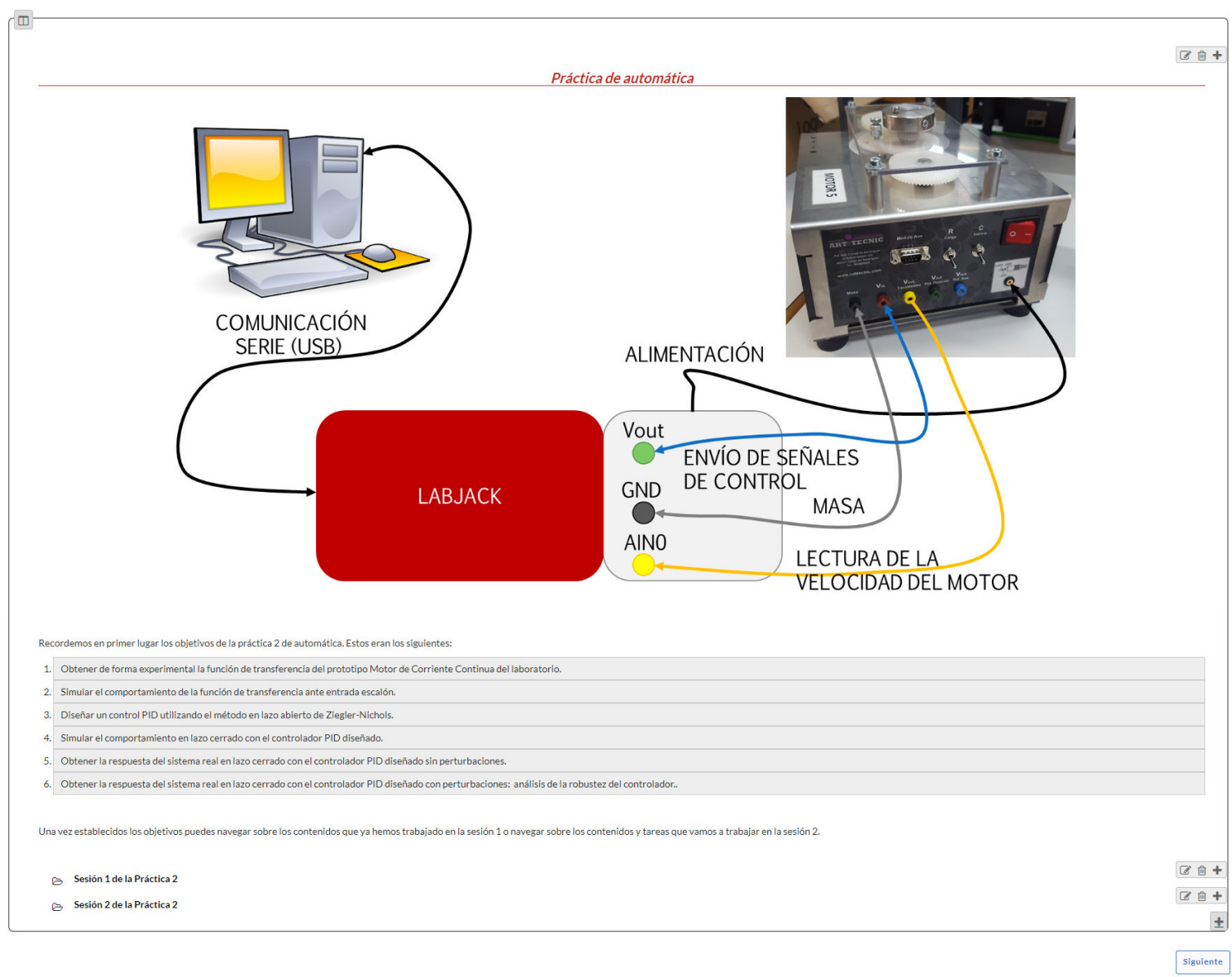

(a) Descripción hardware y objetivos del trabajo a desarrollar.

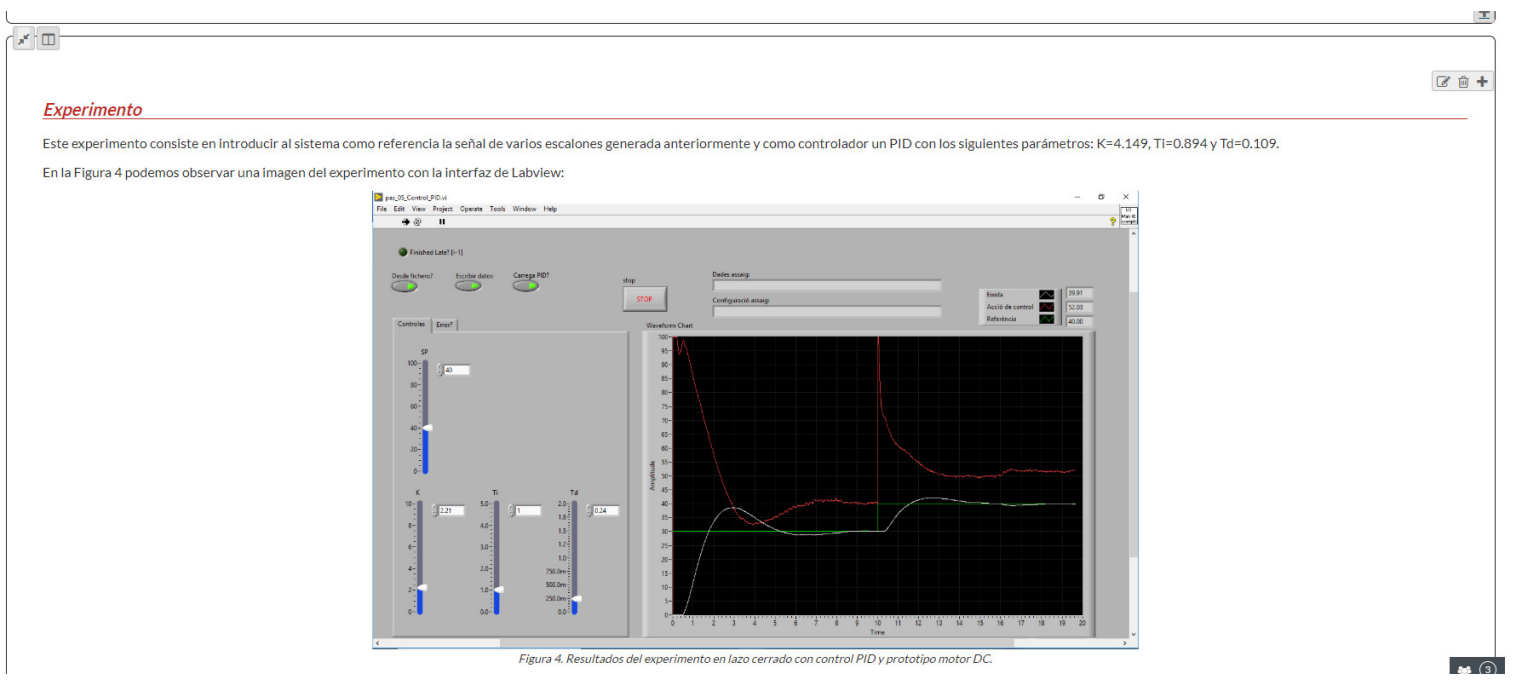

(b) Descripción del software y ejemplo de uso.

Fig. 2: Documentación desarrollada en LESSONS de la plataforma PoliformaT.

2020, Universitat Politècnica de València Congreso IN-RED (2020) 


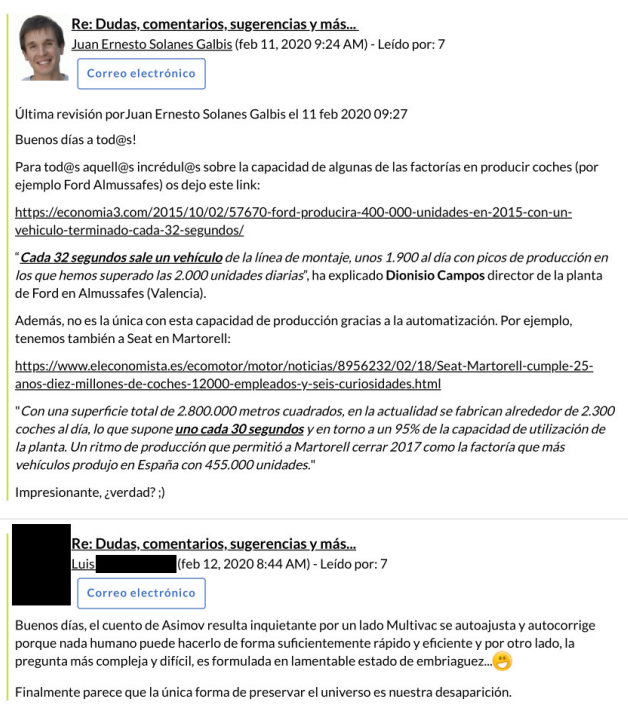

(a)

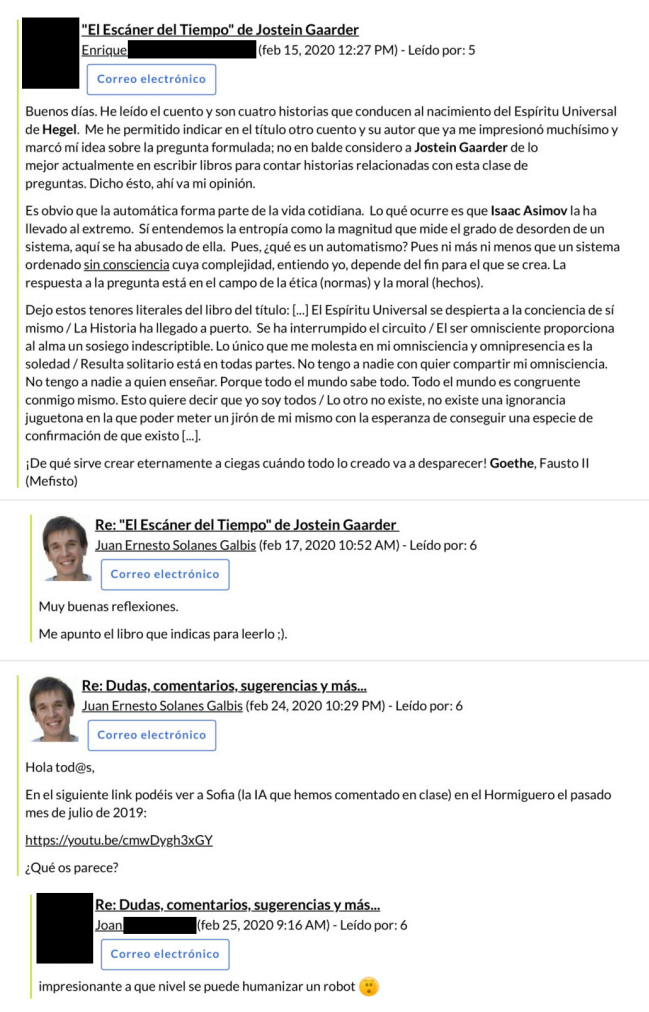

(b)

Fig. 3: Ejemplos de la actividad Foros de la asignatura Electrónica y Automática en el Grado de Ingeniería Mecánica.

\subsubsection{Socialización y motivación del alumnado a través de foros de debate}

De cara a motivar al alumno a lo largo del transcurso de la asignatura y de que dieran su opinión sobre algunos temas relacionados con la mismo, se dispuso de un Foro ubicado en la plataforma PoliformaT (ver Fig. 3). En este foro, el profesor actúa como moderador y propone temas de interés relacionados con la asignatura, como por ejemplo "automática y productividad" o "la robótica actual y futura". El profesor pone links a informaciones en prensa, material multimedia de interés, o artículos cortos o libros, de cara a fomentar el interés del alumno y que vea y analice el alcance de los conceptos que está estudiando en la asignatura. 


\subsection{Métodos empleados}

\subsubsection{Combinación de la modalidad de clase magistral con metodologías activas}

Uno de los principales problema de introducir las metodologías activas propuestas en el apartado anterior es que tienen que combinarse con la modalidad pedagógica de clase magistral sin perjudicar a la cantidad del temario impartido.

Los métodos activos propuestos en las secciones 3.1.3 y 3.1.4 no suponen ningún inconveniente en este sentido, ya que el método de la sección 3.1.3 se realiza en horas de prácticas, y el de la sección 3.1.4 en horas no presenciales.

Pero los métodos de las secciones 3.1.1 y 3.1.2 si que suponen un inconveniente debido a tienen que ser realizados utilizando horas de aula. De cara a mantener la carga del temario, se decide reducir el tiempo empleado por el profesor en la resolución de ejercicios en el aula y dejarlos resueltos en el espacio de la asignatura en la plataforma PoliformaT. De esta forma, el alumno mantiene tanto la carga presencial como los contenidos impartidos en otros cursos, añadiendo trabajo no presencial (resolución de problemas y tutorías) en beneficio de participar activamente en el aula.

\subsubsection{Concienciación al alumnado}

Es importante, a la hora de introducir nuevas propuestas y cambios en las modalidades de enseñanza, que los alumnos sean conocedores de dichos cambios al inicio de la asignatura. Además, también es muy importante proporcionarles todas las rúbricas que el profesorado va a utilizar en los distintos métodos de evaluación.

En este sentido, se hizo un importante esfuerzo al inicio del curso en describir cómo iban a ser las clases y prácticas de aula, además de explicar los cambios realizados en las prácticas de la asignatura y la forma y métodos de evaluación que se iban a utilizar.

\section{Resultados}

El análisis de los resultados de la combinación de la modalidad de clase magistral con las metodologías activas propuestas en la sección 3 se ha realizado desde dos enfoques. Por un lado, se va a analizar el impacto sobre los resultados académicos (examen parcial de automática), comparando el curso 2018/2019 (101 alumnos matriculados), donde se utilizaba la modalidad de clase magistral con prácticas de análisis en simulación, con el curso 2019/2020 (98 alumnos matriculados) con la introducción de la mejora docente propuesta en este artículo. Por otro lado, como forma de conocer la opinión de los alumnos y posibles mejoras a realizar en el futuro, se mostrarán opiniones extraídas de los foros de debate de la asignatura.

El examen parcial de automática consta de dos partes: un test con 10 preguntas teóricas con opción múltiple y una respuesta correcta (30\% de la nota del examen); y dos problemas a desarrollar (70\% de la nota del examen).

La Fig. 4 muestra la comparativa de los resultados académicos de los cursos 2018/2019 y 2019/2020. En la Fig. 4(a) se analiza el porcentaje de alumnos en función de las siguientes categorías: $N O$ PRESENTADOS, los alumnos que no se han presentado al examen parcial de automática; SUS- 
PENDIDO, alumnos que ha obtenido una nota inferior a 5; APROBADO, alumnos que ha obtenido una nota igual o superior que 5 e inferior a 7 ; NOTABLE, alumnos que ha obtenido una nota igual o superior que 7 e inferior a 9; y SOBRESALIENTE, alumnos que ha obtenido una nota superior a 9. Como se aprecia en la figura, el porcentaje de alumnos NO PRESENTADOS se ha mantenido en alrededor el $5 \%$ en ambos cursos. El porcentaje de alumnos que no han aprobado el examen ha pasado del $15 \%$ en el curso 2018/2019 al $3 \%$ en el curso 2019/2020, siendo una mejora muy significativa. El porcentaje de alumnos con una nota igual o superior a 5 e inferior a 7 fue del $36 \%$ en el curso $2018 / 2019$ y del $22 \%$ el curso $2019 / 2020$. El porcentaje de alumnos con una nota igual o superior a 7 e inferior a 9 fue de alrededor del $36 \%$ en el curso 2018/2019 y del $46 \%$ el curso 2019/2020. Por último, el porcentaje de alumnos con una nota superior a 9 fue del $7 \%$ en el curso $2018 / 2019$ y del $23 \%$ el curso $2019 / 2020$.

Del mismo modo, las Fig. 4(b) y 4(c) muestran cómo ha habido una mejora tanto en la desviación estándar (1.68 en el curso 2018/2019 y 1.46 en el curso 2019/2020) como en la media (6.7 en el curso 2018/2019 y 7.8 en el curso 2019/2020) de los resultados obtenidos por los alumnos en el examen parcial de automática.

Por lo que respecta a la opinión de los alumnos, a continuación se exponen los comentarios más comunes:

"Mi experiencia con la asignatura ha sido muy positiva debido a la curva de aprendizaje; de no saber nada a entender mejor el funcionamiento de la automática. Me acuerdo del primer día en que el profesor trajo las gafas de realidad aumentada y todos alucinamos con lo que se podía hacer con ellas. Jordi y yo nos quedamos "flipando" y al salir de clase comentamos el primer día con un compañero que tenía la asignatura convalidada, le dijimos que era la más chula de todas las que estábamos dando.”.

"[...] valoro mucho las prácticas de esta asignatura puesto que al tratarse de algo que no hemos manejado nunca el hecho de poder observar en primera persona, como cambia un sistema según los parámetros que nosotros mismos vamos asignando. Es muy difícil entender algo con lo que no has estado en contacto nunca, por ello pese a nuestra ignorancia en programación, han resultado unas prácticas que han facilitado muchísimo el aprendizaje de la asignatura, cuando ves que un motor reacciona de manera distinta a lo que tu le pides y debes corregir ese comportamiento hasta conseguir lo que uno pretende es cuando realmente entiendes el funcionamiento de un PID.".

"La verdad que la asignatura ha estado muy interesante. Los conceptos al principio son difíciles de ver pero donde verdaderamente entiendes todo es al realizar las prácticas en clase. Ver cómo lo que has estudiado en la teoría coge forma en la práctica. En mi opinión dedicaría mas horas a hacer prácticas o de alguna forma impartir la teoría de forma mas visual como en la práctica del control del motor.".

"Desde un principio no pensaba que llegaríamos hasta poder controlar un motor. La asignatura empezó fuerte al enseñarnos las gafas de realidad virtual, eso me motivo a seguir enganchado a la asignatura.".

"[...] con el juego de las gafas lo pasamos bien y entendimos mejor la palabra control".

(c)) EY-NC-ND 2020, Universitat Politècnica de València

Congreso IN-RED (2020) 


\section{J. Ernesto Solanes y Luis Gracia}

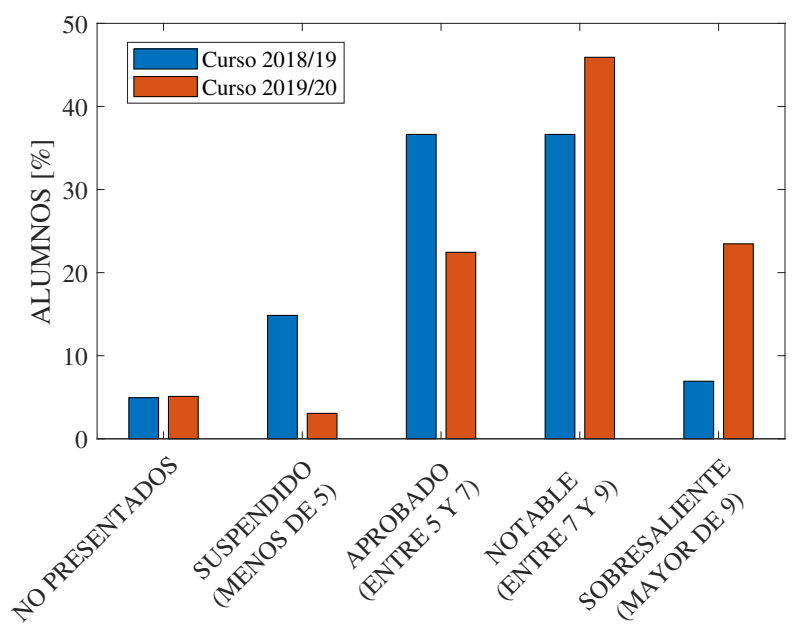

(a) Curso 2018/2019 VS Curso 2019/2020.

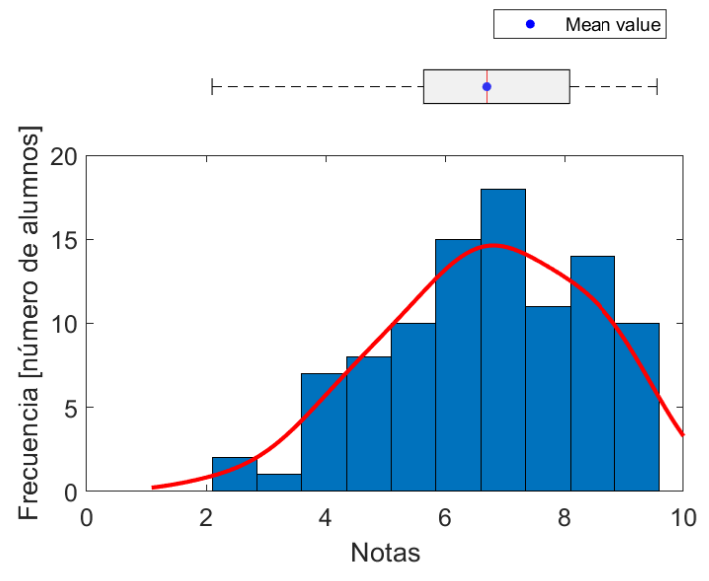

(b) Curso 2018/2019

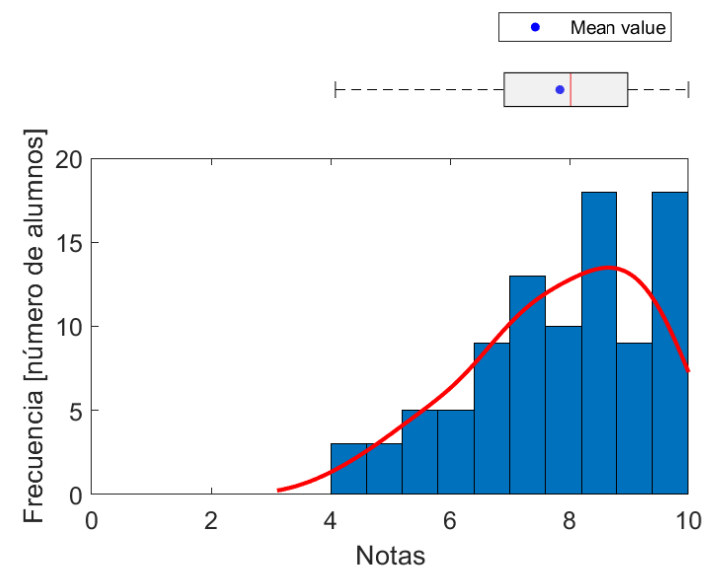

(c) Curso 2019/2020

Fig. 4: Resultados cuantitativos de los cursos 2018/2019 y 2019/2020 de la asignatura Electrónica y Automática de Segundo curso del Grado de Mecánica impartido en la Escuela Politécnica Superior de Alcoy (EPSA). 
"La asignatura ha ido despertando interés en mí a medida que hemos ido avanzando. Al principio, me costaba mucho ver las cosas, pero durante el transcurso de las clases y, sobre todo, de las prácticas de aula, he ido entendiendo las cosas.".

Estos resultados indican que la introducción de metodologías activas ayudan no solamente a mejorar los resultados académicos sino la percepción de los alumnos de lo útiles que resultan los conceptos teóricos que se plantean en esta asignatura, así como su interés y motivación en seguir aprendiendo.

\section{Conclusiones}

En este trabajo se ha descrito y analizado la incorporación de metodologías activas combinadas con la modalidad pedagógica de clase magistral con el fin de mejorar el proceso de enseñanzaaprendizaje en asignaturas con alto contenido teórico de primeros cursos de grado. Concretamente el trabajo describe 4 metodologías incorporadas en la asignatura Electrónica y Automática del Grado de Ingeniería Electrónica de la Universitat Politècnica de València, el balance de resultados académicos obtenidos y opinión de los alumnos.

Por lo que respecta a los resultados, se ha visto que ha habido una mejora importante tanto del número de alumnos aprobados como de la nota media obtenida a raíz de incorporar la propuesta realizada en este trabajo. Si bien solamente se han comparado dos cursos académicos para evitar variabilidad estadística debida a la forma de enseñar que puedan tener profesores distintos, los resultados académicos obtenidos son muy prometedores e invitan a continuar con esta línea de enseñanza. Además, la opinión de los alumnos demuestra que es un procedimiento que les ha ayudado en su proceso de aprendizaje y que están muy satisfechos con la enseñanza recibida.

Finalmente, la experiencia con la propuesta realizada en este trabajo y los resultados obtenidos hacen pensar que la combinación de clases magistrales con metodologías activas puede ser un eslabón necesario de cara a fomentar la actividad del alumno en el aula sin realizar un cambio radical en el modelo pedagógico que, muchas veces no es bien recibido por parte del alumnado, más en los primeros cursos de grado.

\section{Referencias bibliográficas}

Barrows, H. S. (1986). "A taxonomy of problem-based learning methods". En: Medical Education 20.6, págs. 481-486. DOI: $10.1111 / \mathrm{j} .1365-2923.1986 . t b 01386 . x$.

Cerda, Cristian y José L. Saiz (2015). "Aprendizaje autodirigido en estudiantes de pedagogía chilenos: un análisis psicométrico". En: Suma Psicológica 22.2, págs. 129 -136. ISSN: 0121-4381. DOI: https://doi.org/10.1016/j.sumpsi.2015.08.004.

Cervera, M. G. (1999). "Las Tecnologías de la Información y la Comunicación como favorecedoras de los procesos de autoaprendizaje y de formación permanente". En: EDUCAR, págs. 53-60.

Costa, S. S. C. y M. A. Moreira (ene. de 2001). "A resoluÃ §ao de problemas como um tipo especial de apredizagem significativa". En: Caderno Catarinense de Ensino de FÃsica 18(3), págs. 263 -277 . 
Esquivel-Gámez, I. (2014). "Aula Invertida o Modelo Invertido de Aprendizaje: origen, sustento e implicaciones". En: DSAE-Universidad Veracruzana, págs. 143 -160.

Gattullo, Michele y col. (2019). "Towards augmented reality manuals for industry 4.0: A methodology". En: Robotics and Computer-Integrated Manufacturing 56, págs. 276 -286. ISSN: 0736-5845. DOI: $10.1016 / j . r c i m .2018 .10 .001$.

Gómez Paternina, D. A. (2009). "Medios educativos de enseñanza y autonomía del estudiante". En: Studiositas, págs. 39-44.

ISTE (1992). "Guidelines for Accreditation of Educatinal Computing and Technology Programs". En: The International Society for Technology in Education.

Johnson, R.T. y D.W (1994). "An Overview of cooperative learning". En: Thousand, J.; Villa, A,; Nevin, A.

Martínez Costa, Sandra, Finocha Formoso Barro y Antonio Sanjuán Pérez (2017). "Efectos y proceso de la metodología de aprendizaje-servicio en comunicación audiovisual". En: IJERI: International Journal of Educational Research and Innovation 9, págs. 72-89.

Merriam, Sharan B. (1998). Qualitative research and case study applications in education. San Francisco: A joint publication of the Jossey-Bass education series, the Jossey-Bass higher y adult education, pág. 275. ISBN: 0787-910090.

Olaizola, A. (2014). "La clase invertida: usar las TIC para "dar vuelta" a la clase". En: Actas de las X Jornadas de Material Didáctico y Experiencias Innovadoras en Educación Superior, págs. 1 -10 .

Propuestas para la Renovación de las Metodologías Educativas en la Universidad (2006). [Online; accessed 10-October-2019], http://www . catedraunesco.es/archivos/metodologias.pdf.

Proyecto de Competencias Transversales del la Universitat Politècnica de València (2015). [Online; accessed 18-November-2019], https : / / www . upv . es / entidades / ICE / info / Proyecto _ Institucional_CT.pdf.

Sánchez-Vera, Isabel y col. (2018). "Aprendizaje combinado en el aula: clase magistral y peer instruction modificada". En: Congreso In-Red, págs. 1 -14.

Sancho, J.M. (1995). "El medio es el mensaje o el mensaje es el medio? El caso de las Tecnologías de la Información y la Comunicación". En: Revista electrónica Píxel-bit 4(3). 